\title{
Reservoir Development by Waterflooding and Reservoir Performance Analysis
}

\author{
Jiali Li \\ Geological Brigade of the Sixth Oil Production Plant of Daqing Oilfield Co., Ltd., Daqing, Heilongiang, 163453, China.
}

\begin{abstract}
With the rapid development of China's society and economy, relevant industries get good development opportunities, and the demand for oil is increasing. The oil production in the oilfield is increasing day by day. At present, most oilfields gradually enter the middle and late stage of water injection exploitation, which increases the exploitation difficulty and reduces the exploitation amount. In this paper, a comprehensive analysis of waterflooding reservoirs and reservoir performance is made to promote better and faster development of petroleum industry.
\end{abstract}

Keywords: Water injection development, Reservoir, Reservoir performance.

\section{Introduction}

In the process of oil exploitation, water injection can effectively improve the overall quality and efficiency of oil exploitation, and deal with the water injection development technology accordingly. So as to more comprehensively grasp the key methods of reservoir performance analysis, improve the scientific rationality of water injection production technology, and effectively improve the overall level of oilfield production.

\section{Reservoir analysis of water injection development}

According to the analysis of actual oilfield production, it is necessary to ensure the accuracy of experimental information and data, and at the same time have sufficient information, so as to make corresponding accurate judgments on reservoir dynamic analysis. In addition, the relevant analysts need to process the data for the geological model, ensure the information accuracy of the data model, give full play to the advantages and functions of computer technology, start the simulation test on the data model, and then process the oilfield production effect well. In the development process of waterflooding reservoirs, some oil layers are flooded, resulting in poor fluidity, so it is necessary for the mining personnel to quickly drain the internal water and promote the oil wells to resume normal and smooth production [1]. The staff can use the cement test to judge the detailed position of the water layer, timely formulate targeted technical scheme and carry out water injection design, so as to ensure the consistency of water injection effect and water flow direction, and prevent the adverse impact of water outlet on the normal and smooth flow of oil and gas flow. In addition, in the process of water injection reservoir development, it is necessary to carry out reservoir management (as shown in Figure 1), fine description and understanding of oil and gas reservoirs, and adopt perfect and reasonable engineering design and implementation to realize efficient development and production of oil and gas fields. The main feature of reservoir management is multidisciplinary cooperation.

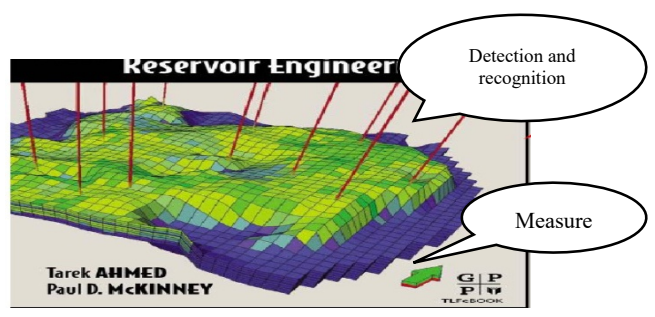

Figure 1 Reservoir operation and management

\section{Dynamic analysis of waterflooding reservoir}

\subsection{Analysis content}

The main content of reservoir dynamic analysis of waterflooding development is the dynamic change of reservoir during waterflooding, understanding and mastering the actual transformation of reservoir during the change process, and grasping the actual situation of reservoir treatment in time. The reservoir development content in water injection system mainly includes the related connection aspects of various reservoirs. Realtime monitoring of the situation and mastering the 
changes of bathing places, such as the internal reservoir and associated water, plays a major role in contacting the related water bodies and triggering the eruption of water bodies in the process of reservoir development. The water body in the reservoir plays an important role in the actual situation of the reservoir. In addition, monitoring the distance between fault and interlayer is conducive to relevant staff to timely control the actual situation in the process of oil reservoir exploitation. The distance between fault and interlayer is very important for the distance of reservoir production. In the actual work, the production personnel need to ensure that they will not touch the block of the interlayer and there will be no landmark collapse and fracture. At the same time, for the development of the reservoir, they need to monitor the relevant information data in real time.

During the exploitation of the reservoir, the production personnel need to timely and accurately grasp the changing data of oil, gas, water and rock. Changing data play a vital role in the whole reservoir production [2]. Staff need to timely, comprehensively and accurately grasp the first-hand information in reservoir development, timely control the oil and gas on the surface, and analyze the connection between disciplines by controlling the problems of various disciplines involved in reservoir development. Reservoir dynamic analysis in waterflooding belongs to a detailed and rigorous attention process, which requires relevant staff to control various information in time. The reservoir development content and related aspects in water injection system involve a lot of information and data in reservoir development. Dynamic reservoir development needs comprehensive and timely control of various information data, so as to avoid a variety of bad problems in reservoir development during water injection development.

\subsection{Basic situation analysis}

In China, the oil field has a relatively complex geology. The chemical and physical properties of the crude oil produced are different from those in the past, and the oil production rate of the oil field is obviously reduced. Therefore, China needs to continuously increase production reserves and research and apply related technologies to enhance oil recovery. In the process of oil production in China, some oil companies are affected by oilfield geology of some multi-layered sandstone reservoirs, resulting in high permeability of crude oil among layers, layers and planes, which has a negative impact on normal and smooth oil production. At the same time, under the condition of serious heterogeneity of the reservoir, there are certain differences in the water absorption of each layer, resulting in the continuous advancement of the water injected into the reservoir under the influence of the high-permeability formation, resulting in great changes in the advancement speed in the vertical and plane, so as to promote the staggered distribution of crude oil and water, resulting in the scattered distribution and mutual accumulation of the remaining crude oil, It further increases the difficulty of oilfield production. In the actual production process of oil field, affected by many factors, injecting a large amount of water into the formation has a great impact on its properties. At the same time, under the condition of long water injection production time, the heterogeneity of reservoir is more serious.

\subsection{Characteristic analysis}

(1)Changes of reservoir fluid properties in waterflooding reservoirs. When oilfield water injection development is carried out for a long time, it is most feared that the salinity of formation water, formation temperature, original composition, pressure in formation and other related information data will change dynamically. For example, during the research of China A Oilfield Company, the water saturation of each pore in the reservoir will be continuously improved by the continuous water injection in the oilfield. At the initial stage of waterflooding development, the pore water saturation showed a significant increase, and with the implementation of waterflooding development in oil fields, its increasing trend gradually decreased in the middle and late stage of exploitation. In addition, the water injection production project of the oilfield causes certain dynamic changes in the salinity of formation water to a certain extent. When fresh water is injected into the formation of the reservoir, the salinity of formation water is continuously reduced with the progress of water injection. When bottom water and edge water are injected into the formation of the reservoir, the substances contained in the water body are similar to those in the oilfield, and the overall mineralization in the formation will not change significantly. When crude oil separated water is injected into the formation, the salinity of water in the formation shows an upward trend.

(2)Dynamic changes of reservoir properties. When people analyze the reservoir properties, they use the model theory to show the reservoir properties to researchers more intuitively. Researchers can use dual media model to show the change of reservoir properties [3]. The water injection pressure of oil field increases gradually with the water injection collection. When the water injection pressure reaches the upper limit of the fracture pressure of the ground layer around the oil well, cracks will occur on the ground surface. After the cracks are restored, the model will still change to a certain extent, which obviously represents the traces of cracks produced by artificial hydraulics. According to the change analysis of well test curve (as shown in Figure 2), the researchers prove that with the continuous water injection, the reservoir will gradually change from homogeneous to composite. At the same time, during the continuous water injection development, the fluid properties of the reservoir will change to a certain extent. The relevant changes in the reservoir are mainly due to the application of water injection production and other methods, which directly leads to the change of reservoir properties, and then leads to a series of conditions. 

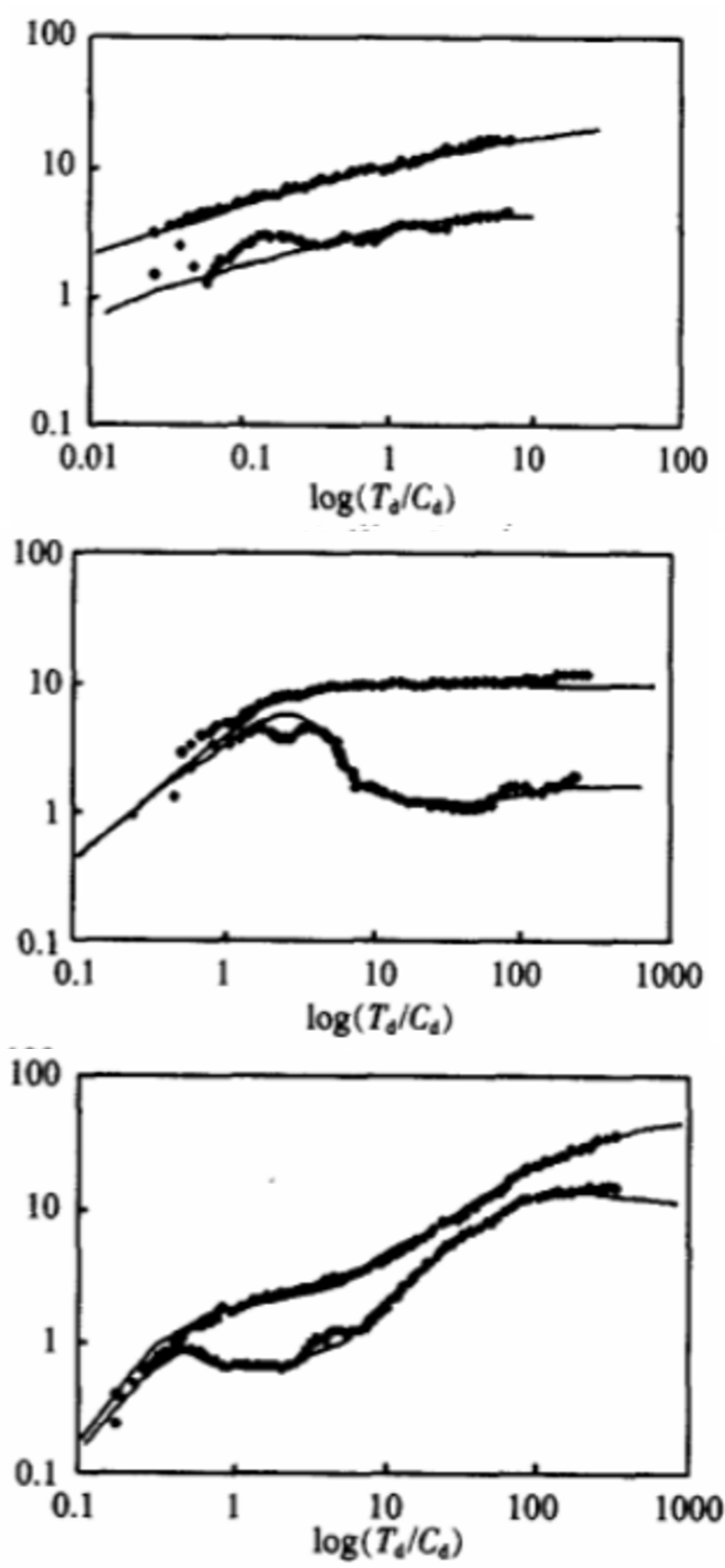

Figure 2 Comparative analysis of the characteristics of the three well test curves of a certain well

(3) Analysis of reservoir parameter variation. In the process of oilfield exploitation by related workers using water injection development technology, the crude oil and mud between formation pores are constantly washed out by the influence of water pressure of water injection, which increases the porosity of oilfield formation as a whole, and at the same time continuously expands the diameter of pore throat, which greatly increases the connectivity between formations and improves the pore throat diameter ratio of formation pores. In addition, under the influence of water injection flow, the mud between pores will be washed away. When accumulation occurs in the formation of the oilfield, it will affect the permeability of the formation of the oilfield, which is not conducive to the smooth exploitation of oil. The rock surface in the reservoir stratum is affected by the water flow, resulting in the continuous reduction of the oil film attached to the surface, increasing the hydrophilicity of the formation rock and the wettability of the oilfield production formation.

(4)Analysis of remaining oil distribution. The distribution of remaining oil in oilfield reservoirs will change correspondingly with the continuous development of oilfield water injection [4]. We analyze according to the distribution area, shape and formation reasons. The remaining oil outside the water injection development area is not washed out by the water flow in the process of exploitation, and most of the remaining oil in the bottom layer presents a flaky distribution. In the process of cluster residual oil exploitation, the permeability channel of reservoir stratum is relatively complicated by geological structure, and the periphery of larger permeability channel mostly contains network-like small permeability channel. In the process of water injection exploitation, part of water flow can not wash out the original small permeability channel, resulting in the original surplus being mostly distributed in the bottom channels. The columnar residual oil is mainly distributed in more than two channels, mainly because the water flow does not pass through the channels during water injection production, or the washed crude oil caused by capillary action in the channels cannot be taken away by the water flow. Angular residual oil is mainly in the corners of the bottom layer and areas where water flow is difficult to contact. Island like residual oil is mainly distributed in the macropores of the reservoir. In water injection production, the crude oil in the center of the pore is difficult to be efficiently exploited, resulting in oil surplus. The film like residual oil is mainly attached to the rock surface, which is mainly due to the insufficient scouring force of water flow in water injection production, which can not effectively overcome the adsorption force between rock and oil film to produce crude oil surplus.

\subsection{Analytical method}

(1)Numerical simulation analysis method. With the continuous progress and development of China's scientific informationization, numerical simulation analysis method is widely used in the dynamic analysis of oil reservoirs, mainly relying on computer software to effectively and dynamically simulate the development process of oilfield water injection, which is more convenient for researchers to formulate scientific and reasonable oil exploitation plans according to the actual situation of the model [5]. In the process of numerical simulation, researchers need to comprehensively collect a number of information data of the oilfield, create a targeted geological model with data, and divide the model into cells. The whole production process is simulated by the operation of computer software, and the dynamic changes of multiple data of oil reservoirs during exploitation are comprehensively analyzed.

(2)Well test analysis method. The staff used this method to analyze the performance of waterflooding reservoirs, and effectively analyzed the dynamic changes of corresponding data by relying on the relevant data such as 
oilfield pressure, temperature and production measured by well testing operations. At the same time, the staff scientifically and reasonably applied the seepage mechanics theory, analyzed and collected information data, effectively obtained the production status, actual productivity and physical property data of oilfield water injection development, and comprehensively analyzed the oil fluid communication in sandstone layer.

(3)Empirical formula method for plate analysis. This analysis method is mainly used by staff to analyze the changes of oil and gas properties in strata and predict the changes of many parameters, which belongs to the dynamic analysis method of reservoir parameters. Workers need to analyze relevant data in practical production. In the process of measuring and extracting oilfield oil and gas parameters, practical operation is simple, which improves the practical effectiveness of this method. In the process of applying the empirical formula method of chart analysis, the staff provide a large amount of information data collected by the chart method as the basis of data analysis, and then comprehensively analyze and summarize the actual data, determine a set of empirical formula, and use the formula to calculate the relevant data. During the dynamic analysis of water injection reservoir, the staff collected and sorted out various data, and selected appropriate analysis methods according to the characteristics of data change, so as to improve the actual effect of water injection development.

\section{Conclusions}

In the process of continuous water injection development of the reservoir, many data of the reservoir change to varying degrees, which has a certain impact on the followup oil production. Therefore, relevant personnel need to adopt appropriate reservoir dynamic analysis methods for waterflooding development, comprehensively analyze the distribution of remaining oil, understand its change characteristics, comprehensively improve the quality and efficiency of waterflooding, and obtain more comprehensive benefits.

\section{References}

1. Liu Cong. Dynamic analysis of water injection development reservoirs[J]. Chemical Engineering and Equipment, 2020(1):141-141.

2. Jiang Xueying. Analysis of dynamic analysis of waterflooding development reservoir[J]. Chemical Engineering and Equipment, 2019, 000(003):171171.

3. $\mathrm{Xu}$ Maohe. Discussion on dynamic analysis of waterflooding development reservoir[J]. Chemical Industry Management, 2019(21):224-224.

4. $\mathrm{Pu}$ Menglei. Discussion on dynamic analysis of waterflooding development reservoir[J]. Chemical Management, 2019, 000(026):213-214.

5. Wang Rui. Research on the dynamic analysis method of waterflooding development reservoir[J]. China Petroleum \& Petrochemical, 2019, 09(No.368):91-92. 